$5-9-2008$

\title{
Synthesis of Substituted 2-Amino-Cyclobutanones
}

\author{
Nicola Armoush \\ Loyola University Chicago \\ Preeti Syal \\ Daniel P. Becker \\ Loyola University Chicago, dbecke3@luc.edu
}

Follow this and additional works at: https://ecommons.luc.edu/chemistry_facpubs

Part of the Chemistry Commons

\section{Author Manuscript}

This is a pre-publication author manuscript of the final, published article.

\section{Recommended Citation}

Armoush, Nicola; Syal, Preeti; and Becker, Daniel P.. Synthesis of Substituted 2-Amino-Cyclobutanones. Synthetic Communications, 38, 11: , 2008. Retrieved from Loyola eCommons, Chemistry: Faculty Publications and Other Works, http://dx.doi.org/10.1080/00397910801981110

This Article is brought to you for free and open access by the Faculty Publications and Other Works by Department at Loyola eCommons. It has been accepted for inclusion in Chemistry: Faculty Publications and Other Works by an authorized administrator of Loyola eCommons. For more information, please contact ecommons@luc.edu. (c) (i) $\odot$

This work is licensed under a Creative Commons Attribution-Noncommercial-No Derivative Works 3.0 License. (c) 2008 Taylor \& Francis 


\title{
Synthesis of Substituted 2-Amino-Cyclobutanones
}

\author{
Nicola Armoush, Preeti Kataria and Daniel P. Becker* \\ Loyola University Chicago, 6525 North Sheridan Road, Chicago, IL 60626
}

\begin{abstract}
A series of weakly nucleophilic nitrogen derivatives including carbamates, amides, sulfonamides and anilines were reacted with 1,2bis(trimethylsilyloxy)cyclobutene under acidic conditions to afford various substituted 2aminocyclobutanone derivatives 3a-i in modest to excellent yields.
\end{abstract}

Keywords: cyclobutene, cyclobutanone, 2-aminocyclobutanone

Cyclobutanones are important molecules both as synthetic targets and as synthetic intermediates with unique and useful applications. ${ }^{[1,2]}$ Several natural products contain a cyclobutanone moiety, including the diterpenes acetylcoriacenone and isoacetylcoriacenone, isolated from the brown sea algae Pachydictyon coriaceum, and the monoterpene (1S,5S)-filifolone from the Arizona sand sage Artemisia filifolia, and (1R,5R)-filifolone from the Australian sandfly bush Ziera smithii, and chrysanthenone from the flowers of Chrysanthemum sinese. ${ }^{[3]}$ The four-membered ring endows cyclobutanones with a degree of conformational rigidity, and the strain inherent in the ring makes the ketone carbonyl more electrophilic than an unstrained ketone. Cyclobutanones have been reported to exhibit enzyme inhibitory activity toward betalactamase $^{[4]}$ and the serine protease, elastase. ${ }^{[5]}$ Fairlie has shown that protease inhibitors 
recognize substrate segments in the beta-sheet conformation, ${ }^{[6,7]}$ and Burgess has demonstrated that cis-1,3-disubstituted cyclobutane amino acid derivatives adopt an extended, beta-sheet conformation, ${ }^{[8]}$ which suggests that more highly functionalized cyclobutanones may serve as potent and selective protease inhibitors. The employment of small molecule peptidomimetic derivatives to mimic peptide conformational ensembles is critical in drug discovery toward the development of new pharmaceuticals, ${ }^{[9]}$ yet the cyclobutanone moiety is underutilized in medicinal chemistry because methods for the preparation of more adaptable and highly substituted cyclobutanones are limited. The synthesis of highly substituted cyclobutanones is a topic of current interest which has recently been addressed by Doyle ${ }^{[10]}$ utilizing a carbene insertion approach to prepare 3-aryl-2-silyloxy-2-carbomethoxy cyclobutanones. Thus, cyclobutanones are very important targets and intermediates, yet methods for the preparation of more highly substituted cyclobutanones are limited.

We required protected 2-amino cyclobutanone derivatives to serve as intermediates toward the preparation of peptidomimetic cyclobutanones. Vederas reported the synthesis of 2-carbobenzyloxyaminocyclobutanone 2 via the reaction of 1,2bis(trimethylsilyloxy)cyclobutene with benzyl carbamate in the presence of $\mathrm{HCl}$ in $81 \%$ yield (Scheme 1). ${ }^{[1]}$ The requisite 1,2-bis(trimethylsilyloxy)cyclobutene is in turn prepared via the acyloin cyclization of dimethyl succinate in the presence of trimethylsilyl chloride as described by Frahm. ${ }^{[12]}$ For our related studies toward the preparation of more highly functionalized cyclobutanones we required $\mathrm{N}$-alkylated 2aminocyclobutanones and derivatives with alternate protecting groups. Nucleophilic secondary amines have also been reported to afford N,N-disubstituted 2- 
aminocyclobutanones under neutral conditions. ${ }^{[13,14]}$ We questioned if other poorly nucleophilic amine derivatives would similarly react with the cyclobutene. Thus, we undertook an exploration of the scope of the reaction of 1,2bis(trimethylsilyloxy)cyclobutene with other poorly-nucleophilic nucleophiles and found that amides and sulfonamides in addition to more hindered secondary carbamates react efficiently with cyclobutene 1 to afford protected 2-aminocyclobutanone derivatives.

The results of our studies are summarized in Table 1. We have explored several sets of conditions for the reaction of N-nucleophiles with 1,2bis(trimethylsilyloxy)cyclobutene and have found that reflux in $1 \mathrm{M} \mathrm{HCl}$ in ether affords the most general and reproducible results. $\mathrm{N}$-alkyl benzyl carbamates were employed to access the N-alkylated cyclobutanone derivatives. N-benzyl benzylcarbamate affords cyclobutanone 3a in similar yield (60\%) to the unsubstituted parent (69\% in our hands), thus the reaction does not suffer substantially from the additional steric demand. Nmethyl benzylcarbamate reacted efficiently as well to give $\mathbf{3 b}$, although it was quite difficult to isolate the product cyclobutanone from the carbamate starting material, although numerous TLC solvent systems were explored, hence the yield of the N-methyl derivative is somewhat lower (48\%). Given the poor nucleophilicity of the carbamate nitrogen, we were encouraged to explore sulfonamide derivatives. We were very pleased that p-toluenesulfonamide reacts in very high yield (90\%) to afford $\mathbf{3 c}$, and the N-methyl p-toluenesulfonamide affords the corresponding N-methyl-2-aminocyclobutanone sulfonamide $\mathbf{3 d}$ in $80 \%$ yield. We then turned our attention to amides as nucleophilic partners in the reaction. Although the reaction is successful with phenyl acetamide affording cyclobutanone 3e, the yield is lower (39\%). Benzamide, on the other hand, 
gave a higher yield of cyclobutanone benzamide $3 \mathbf{f}(68 \%)$ comparable to the unsubstituted and N-benzyl carbamate derivatives. 4-Chlorobenzoic acid gave a lower yield of cyclobutanone benzamide $3 \mathrm{~g}$ (42\%) presumably due to lower electron density on the amide nitrogen. Reaction with the more sterically demanding and electron-poor 2chlorobenzene gave only very poor yields of cyclobutanone adduct $\mathbf{3 h}(10 \%)$ which was difficult to purify and characterize. Reaction with 4-cyanoaniline to afford cyclobutanone $3 \mathbf{i}$ proceeded in low yield (26\%) but was gratifying in contrast to the attempted reactions under acidic conditions with aniline or N-methyl aniline which did not afford any cyclobutanone adducts. The difference in reactivity is presumably due to protonation of the aniline and N-methyl aniline substrates, whereas 4-cyanoaniline is significantly less basic.

In summary, the scope of the reaction of 1,2-bis(trimethylsilyloxy)cyclobutene with various nitrogen nucleophiles under acid conditions has been explored. Sulfonamides gave high yields of cyclobutanone adducts, and carbamates gave generally good yields. Reaction with benzamide proceeded well, but lower yields were obtained when employing substituted benzamides or an alkyl amide. Several anilines failed to provide cyclobutanone adducts with the exception of 4-cyanoaniline which afforded the $\mathrm{N}$-aryl-2-aminocyclobutanone adduct in lower yield.

\section{Experimental Section}

All reactions were performed under an atmosphere of nitrogen, and all solvents and reagents were used without further purification unless otherwise noted. Merck silica gel 
60 (230-400 mesh) was used for flash chromatography. Merck Kieselgel 60 F254 DCFertigplatten (0.25 mm, Art. 5719) were used for TLC. ${ }^{1} \mathrm{H}$ NMR spectra were obtained from either a Varian INOVA 300 or Varian Gemini 2000300 MHz spectrometer with tetramethylsilane (TMS) as an internal standard. Noise-decoupled and ${ }^{13} \mathrm{C}$ NMR spectra were recorded at $75 \mathrm{MHz}$ on either the Varian INOVA 300 or Varian Gemini 2000 spectrometer. IR spectra were recorded on a Thermo Nicolet Nexus 670 FT-IR. Mass spectra were run on a Thermo Finnigan LCQ Advantage instrument. 1,2Bis(trimethylsilyloxy)cyclobutene was prepared according to the procedure of Frahm. ${ }^{[12]}$

\section{Benzyl benzyl(2-oxocyclobutyl)carbamate (3a). To a solution of N-benzyl} benzylcarbamate $^{[15]}(150 \mathrm{mg}, 0.62 \mathrm{mmol})$ in $1.0 \mathrm{M} \mathrm{HCl}$ solution in diethyl ether $(3 \mathrm{~mL})$ cooled to $0^{\circ} \mathrm{C}$ was added 1,2-bis(trimethylsilyloxy)cyclobutene (143 $\mathrm{mg}, 0.62 \mathrm{mmol}$ ) dropwise over $5 \mathrm{~min}$. The reaction was then allowed to reflux for $4 \mathrm{~h}$ at $80^{\circ} \mathrm{C}$. Concentration gave a residue which was purified on silica gel eluting with $\mathrm{Et}_{2} \mathrm{O} / \mathrm{h}$ exane (1/1) to afford the desired carbamate-cyclobutanone 3a (110 mg, 58\%) as a pale yellow oil. FT-IR (thin film) 3054, 1794, 1697, $1421 \mathrm{~cm}^{-1}$; ${ }^{1} \mathrm{H}$ NMR (300 MHz, $\mathrm{CDCl}_{3}$ ) $\delta$ 7.36$7.20(10 \mathrm{H}, \mathrm{m}), 5.15(1 \mathrm{H}, \mathrm{m}), 4.56-4.38(4 \mathrm{H}, \mathrm{m}), 2.81-2.52(2 \mathrm{H}, \mathrm{m}), 2.34-2.05(2 \mathrm{H}, \mathrm{m})$; ${ }^{13} \mathrm{C}$ NMR $\left(75 \mathrm{MHz}, \mathrm{CDCl}_{3}\right) \delta 205.3,178.1,128.8,128.6,128.2,128.0,127.6,127.1$, 67.9, 51.8, 41.5, 40.9, 18.4. MS m/z (\% rel. int.) $309.07\left(40, \mathrm{M}^{+}\right), 219.27$ (100, Mbenzyl).

Benzyl methyl(2-oxocyclobutyl)carbamate (3b). To a solution of N-methyl carbamate $^{[16]}(175 \mathrm{mg}, 1.06 \mathrm{mmol})$ in $1.0 \mathrm{M} \mathrm{HCl} / \mathrm{ether}(12 \mathrm{ml})$ was added 1,2-bis 
(trimethlysilyloxy)cyclobutene $(405 \mathrm{mg}, 1.7 \mathrm{mmol})$ at $0^{\circ} \mathrm{C}$. The reaction mixture was heated at $55^{\circ} \mathrm{C}$ for four hours, then the solvent was removed under vacuum.

Chromatography on silica gel eluting with $2 / 98 \mathrm{i}-\mathrm{PrOH} / \mathrm{CH}_{2} \mathrm{Cl}_{2}$ provided benzyl methyl(2-oxocyclobutyl)carbamate $\mathbf{3 b}$ as an oil (118 mg, 48\%). IR (thin film) 1792, $1697 \mathrm{~cm}^{-1} ;{ }^{1} \mathrm{H}$ NMR $\left(300 \mathrm{MHz}, \mathrm{CDCl}_{3}\right) \delta 7.35(5 \mathrm{H}, \mathrm{m}), 5.13(2 \mathrm{H}, \mathrm{s}), 5.10(1 \mathrm{H}, \mathrm{m})$, $2.90(3 \mathrm{H}, \mathrm{s}), 2.81(2 \mathrm{H}, \mathrm{m}), 2.48(1 \mathrm{H}, \mathrm{m}), 2.33(1 \mathrm{H}, \mathrm{m}) ;{ }^{13} \mathrm{C} \mathrm{NMR}\left(75 \mathrm{MHz}, \mathrm{CDCl}_{3}\right)$ $\delta 205.1,195.5,155.7,136.3,136.0,129.2,128.6,111.7,71.2,67.8,41.2,33.1,17.1 ;$ MS $\mathrm{m} / \mathrm{z}=91$ (tropylium ion), $\mathrm{m} / \mathrm{z}=98\left(\mathrm{M}^{+}-135, \mathrm{M}-\mathrm{Cbz}\right.$ group$), \mathrm{m} / \mathrm{z}=191\left(\mathrm{M}^{+}-42\right.$, loss of ketene), $\mathrm{m} / \mathrm{z}=205\left(\mathrm{M}^{+}-28\right.$, loss of $\left.\mathrm{CO}\right), \mathrm{m} / \mathrm{z}=233\left(\mathrm{M}^{+}\right)$.

4-Methyl- $N$-(2-oxocyclobutyl)benzenesulfonamide (3c). To a solution of 4methylbenzenesulfonamide $(111 \mathrm{mg}, 0.65 \mathrm{mmol})$ in $1.0 \mathrm{M} \mathrm{HCl}$ solution in diethyl ether ( $3 \mathrm{~mL}$ ) cooled to $0^{\circ} \mathrm{C}$ was added 1,2-bis(trimethylsilyloxy)cyclobutene $(150 \mathrm{mg}, 0.65$ mmol) dropwise over $5 \mathrm{~min}$. The reaction was then allowed to reflux for $4 \mathrm{~h}$ at $80^{\circ} \mathrm{C}$. Concentration gave a residue which was purified on silica gel eluting with EtOAc/toluene (10/90) to afford the desired sulfonamide-cyclobutanone $3 \mathbf{c}(140 \mathrm{mg}, 90 \%)$ as a white crystalline solid. FT-IR (KBr) 3582, 3054, 1791, 1421, $896 \mathrm{~cm}^{-1}$; ${ }^{1} \mathrm{H}$ NMR $(300 \mathrm{MHz}$, $\left.\mathrm{CDCl}_{3}\right) \delta 7.77(2 \mathrm{H}, \mathrm{d}, \mathrm{J}=8.2 \mathrm{~Hz}), 7.32(2 \mathrm{H}, \mathrm{d}, \mathrm{J}=8.1 \mathrm{~Hz}), 5.04(1 \mathrm{H}, \mathrm{d}, \mathrm{J}=7.9 \mathrm{~Hz}), 4.69$ $(1 \mathrm{H}, \mathrm{q}, \mathrm{J}=8.6 \mathrm{~Hz}), 3.00-2.71(2 \mathrm{H}, \mathrm{m}), 2.44(3 \mathrm{H}, \mathrm{t}, \mathrm{J}=5.1 \mathrm{~Hz}), 2.37(1 \mathrm{H}, \mathrm{m}), 1.81$ $(1 \mathrm{H}, \mathrm{m}) ;{ }^{13} \mathrm{C} \mathrm{NMR}\left(75 \mathrm{MHz}, \mathrm{CDCl}_{3}\right) \delta 207.8,137.6,133.9,129.2,118.3,62.9,46.1,35.8$, 29.8. MS m/z (\% rel. int.) 238.47 (25, M-1), 219.27 (100, M-benzyl). 
$N, 4-D i m e t h y l-N$-(2-oxocyclobutyl)benzenesulfonamide (3d). To a solution of Nmethyl-p-toluene sulfonamide (144 mg, $0.78 \mathrm{mmol})$ in $1.0 \mathrm{M} \mathrm{HCl}$ /ether $(3 \mathrm{ml})$ was added 1,2-bis(trimethylsilyloxy)cyclobutene $(160 \mathrm{mg}, 0.60 \mathrm{mmol})$ at $0^{\circ} \mathrm{C}$. The reaction mixture was heated at $55^{\circ} \mathrm{C}$ for 3.5 hours, then the solvent was removed under vacuum. Chromatography on silica gel eluting with 1/99 ethyl acetate/dichloromethane provided N,4-dimethyl -N-(2-oxocyclobutyl)benzenesulfonamide $\mathbf{3 d}$ as an oil (123 mg, 80\%). IR (thin film) 1790, $1513 \mathrm{~cm}^{-1} ;{ }^{1} \mathrm{H} \mathrm{NMR}\left(300 \mathrm{MHz}, \mathrm{CDCl}_{3}\right) \delta 7.71(2 \mathrm{H}, \mathrm{d}, J=8.24 \mathrm{~Hz})$, $7.30(2 \mathrm{H}, \mathrm{d}, J=7.96 \mathrm{~Hz}), 5.34(1 \mathrm{H}, \mathrm{t}, J=8.7,10.7 \mathrm{~Hz}), 2.88-2.78(2 \mathrm{H}, \mathrm{m}), 2.70(3 \mathrm{H}, \mathrm{s})$, $2.64(3 \mathrm{H}, \mathrm{s}), 2.23(1 \mathrm{H}, \mathrm{dd}, J=4.6,10.7 \mathrm{~Hz}), 1.97(1 \mathrm{H}, \mathrm{m}) ;{ }^{13} \mathrm{C} \mathrm{NMR}\left(75 \mathrm{MHz}, \mathrm{CDCl}_{3}\right) \delta$ 204.0, 144.1, 135.9, 130.0, 127.3, 71.4, 41.9, 31.0, 22.1, 16.2; MS m/z =91 (tropylium ion), $\mathrm{m} / \mathrm{z}=155\left(\mathrm{M}^{+}-98\right.$, loss of Me-N-cyclobutanone $), \mathrm{m} / \mathrm{z}=225\left(\mathrm{M}^{+}-28\right.$, loss of $\left.\mathrm{CO}\right)$, $\mathrm{m} / \mathrm{z}=211\left(\mathrm{M}^{+}-42\right.$, loss of ketene $), \mathrm{m} / \mathrm{z}=254\left(\mathrm{M}^{+}+1\right)$

N-(2-Oxocyclobutyl)-2-phenylacetamide (3e). To a solution of phenylacetamide (150 $\mathrm{mg}, 1.11 \mathrm{mmol})$ in $1.0 \mathrm{M} \mathrm{HCl}$ /ether $(3 \mathrm{ml})$ and dichloromethane $(2 \mathrm{ml})$ was added 1,2-bis (trimethylsilyloxy)cyclobutene $(255 \mathrm{mg}, 1.11 \mathrm{mmol})$ at $0^{\circ} \mathrm{C}$. The reaction mixture was heated at $55^{\circ} \mathrm{C}$ for 3.5 hours, then the solvent was removed under vacuum. Chromatography on silica gel eluting with 30/70 ethyl acetate/dichloromethane provided N-(2-oxocyclobutyl)-2-phenylacetamide $3 \mathbf{e}$ as an oil (88 mg, 39\%). IR (thin film) 3274, 1790.2, $1647 \mathrm{~cm}^{-1} ;{ }^{1} \mathrm{H}$ NMR (300 MHz, $\left.\mathrm{CDCl}_{3}\right) \delta 7.23-7.37(5 \mathrm{H}, \mathrm{m}), 5.38(1 \mathrm{H}, \mathrm{s}), 5.19$ $(2 \mathrm{H}, \mathrm{s}), 4.78-4.87(1 \mathrm{H}, \mathrm{q}, J=7.91,8.05 \mathrm{~Hz}), 2.86(2 \mathrm{H}, \mathrm{m}), 2.29-2.41(1 \mathrm{H}, \mathrm{m}), 1.95-2.08$ $(1 \mathrm{H}, \mathrm{m}) ;{ }^{13} \mathrm{C} \mathrm{NMR}\left(75 \mathrm{MHz}, \mathrm{CDCl}_{3}\right) \delta 205.9,171.3,134.5,129.6,127.7,64.4,43.3$, 42.2, 19.6; MS: $\mathrm{m} / \mathrm{z}=91.1$ (tropylium ion), $\mathrm{m} / \mathrm{z}=119\left(\mathrm{M}^{+}-84\right.$, loss of $\mathrm{N}-\mathrm{H}$ 
cyclobutanone), $\mathrm{m} / \mathrm{z}=161\left(\mathrm{M}^{+}-42\right.$, loss of ketene $), \mathrm{m} / \mathrm{z}=175\left(\mathrm{M}^{+}-28\right.$, loss of $\left.\mathrm{CO}\right), \mathrm{m} / \mathrm{z}$ $=202\left(\mathrm{M}^{+}-1\right)$.

$N$-(2-Oxocyclobutyl)benzamide (3f). To a solution of benzamide (78.7 $\mathrm{mg}, 0.65 \mathrm{mmol}$ ) in $1.0 \mathrm{M} \mathrm{HCl}$ solution in diethyl ether $(3 \mathrm{~mL})$ cooled to $0^{\circ} \mathrm{C}$ was added $1,2-$ bis(trimethylsilyloxy)cyclobutene $(150 \mathrm{mg}, 0.65 \mathrm{mmol})$ dropwise over $5 \mathrm{~min}$. The reaction was then allowed to reflux for $4 \mathrm{~h}$ at $80^{\circ} \mathrm{C}$. Concentration gave a residue which was purified on silica gel eluting with EtOAc/toluene (15/85) to afford the desired benzamide-cyclobutanone $\mathbf{3 f}$ ( $84 \mathrm{mg}, 68 \%$ ) as a white crystalline solid. FT-IR (KBr) $3582,3054,1791,1665,1421 \mathrm{~cm}^{-1}$; MS ${ }^{1} \mathrm{H}$ NMR $\left(300 \mathrm{MHz}, \mathrm{CDCl}_{3}\right) \delta 7.76(2 \mathrm{H}, \mathrm{d}, \mathrm{J}=$ $6.9 \mathrm{~Hz}), 7.51(2 \mathrm{H}, \mathrm{t}, \mathrm{J}=7.4 \mathrm{~Hz}), 7.41(1 \mathrm{H}, \mathrm{t}, \mathrm{J}=7.3 \mathrm{~Hz}), 6.87(1 \mathrm{H}, \mathrm{d}, \mathrm{J}=6.7 \mathrm{~Hz}), 5.15$ $(1 \mathrm{H}, \mathrm{q}, \mathrm{J}=8.5 \mathrm{~Hz}), 3.01(2 \mathrm{H}, \mathrm{t}, \mathrm{J}=8.7 \mathrm{~Hz}), 2.52(1 \mathrm{H}, \mathrm{m}), 2.18(1 \mathrm{H}, \mathrm{m}) ;{ }^{13} \mathrm{C} \mathrm{NMR}(75$ $\left.\mathrm{MHz}, \mathrm{CDCl}_{3}\right) \delta 205.7,167.0,133.1,132.7,128.7,127.2,64.6,42.3,19.8 . \mathrm{m} / \mathrm{z}(\%$ rel. int.) $189.13\left(55, \mathrm{M}^{+}\right), 144.13(65, \mathrm{M}-\mathrm{NHCO}), 105.07\left(100, \mathrm{M}-\mathrm{NHCHCOCH}_{2} \mathrm{CH}_{2}\right)$.

4-Chloro- $N$-(2-oxocyclobutyl)benzamide (3g). To a solution of 4-chlorobenzamide (100 mg, $0.64 \mathrm{mmol})$ in $\mathrm{HCl} /$ ether $(2 \mathrm{ml})$ and THF $(4 \mathrm{ml})$ was added 1,2-bis (trimethylsilyloxy)cyclobutene $(230 \mathrm{mg}, 1.00 \mathrm{mmol})$ at $0^{\circ} \mathrm{C}$. The reaction mixture was heated at $80^{\circ} \mathrm{C}$ for 3.5 hours, then the solvent was removed under vacuum. Chromatography on silica gel eluting with 2/98 ethyl acetate/dichloromethane provided 4-chloro-N-(2-oxocyclobutyl)benzamide $\mathbf{3 g}$ as a solid (60 mg, 42\%). IR (thin film) 2253, 1792, $1662 \mathrm{~cm}^{-1} ;{ }^{1} \mathrm{H}$ NMR (300 MHz, $\left.\mathrm{CDCl}_{3}\right) \delta 7.69(2 \mathrm{H}, \mathrm{d}, J=2.2 \mathrm{~Hz}), 7.38(2 \mathrm{H}, \mathrm{d}, J=$ $4.8 \mathrm{~Hz}), 6.84(1 \mathrm{H}, \mathrm{s}), 5.14(1 \mathrm{H}, \mathrm{m}), 3.02(2 \mathrm{H}, \mathrm{m}), 2.57-2.49(1 \mathrm{H}, \mathrm{m}), 2.22-2.14(1 \mathrm{H}, \mathrm{m})$; 
${ }^{13} \mathrm{C}$ NMR $\left(75 \mathrm{MHz}, \mathrm{CDCl}_{3}\right) \delta 206.0,166.0,161.0,138.0,132.0,129.0,64.6,42.3,19.7$;

MS: $\mathrm{m} / \mathrm{z}=111\left(\mathrm{M}^{+}-112\right.$ loss of chlorobenzyl), $\mathrm{m} / \mathrm{z}=139\left(\mathrm{M}^{+}-84\right.$, loss of H-N-

cyclobutanone), $\mathrm{m} / \mathrm{z}=181\left(\mathrm{M}^{+}-42\right.$, loss of ketene $), \mathrm{m} / \mathrm{z}=223\left(\mathrm{M}^{+}\right)$.

2-Chloro- $N$-(2-oxocyclobutyl)benzamide (3h). To a solution of 2-chlorobenzamide (260 mg, $1.6 \mathrm{mmol})$ in $1.0 \mathrm{HCl} /$ ether $(3 \mathrm{ml})$ and dichloromethane $(3 \mathrm{ml})$ was added 1,2bis (trimethlysilyloxy)cyclobutene $(384 \mathrm{mg}, 1.6 \mathrm{mmol})$ at $0^{\circ} \mathrm{C}$. The reaction mixture was heated at $80^{\circ} \mathrm{C}$ for thr3.5 hours, then the solvent was removed under vacuum.

Chromatography on silica gel eluting with 10/90 ethyl acetate/dichloromethane provided 2-chloro-N-(2-oxocyclobutyl)benzamide $\mathbf{3 h}$ as a white solid (40 mg, $10 \%$ ). FT-IR (thin film) $2253,1793,1666 \mathrm{~cm}^{-1} ;{ }^{1} \mathrm{H} \mathrm{NMR}\left(300 \mathrm{MHz}, \mathrm{CDCl}_{3}\right) \delta 7.62(1 \mathrm{H}, \mathrm{d}, J=1.1 \mathrm{~Hz})$, $7.39-7.08(3 \mathrm{H}, \mathrm{m}), 7.08(1 \mathrm{H}, \mathrm{s}), 5.14(1 \mathrm{H}, \mathrm{q}, J=10 \mathrm{~Hz}), 3.04-2.91(2 \mathrm{H}, \mathrm{m}), 2.53-2.50$ $(1 \mathrm{H}, \mathrm{m}), 2.22-2.04(1 \mathrm{H}, \mathrm{m}) ;{ }^{13} \mathrm{C} \mathrm{NMR}\left(75 \mathrm{MHz}, \mathrm{CDCl}_{3}\right) \delta 205.1,166.3,133.9,131.9$, $131.0,130.5,128.9,127.3,64.7,42.5,19.7 ; \mathrm{MS} \mathrm{m} / \mathrm{z}=91$ (tropylium ion), $\mathrm{m} / \mathrm{z}=139$ $\left(\mathrm{M}^{+}-84\right.$, loss of H-N-cyclobutanone $), \mathrm{m} / \mathrm{z}=224\left(\mathrm{M}^{+}+1\right)$.

4-[(2-Oxocyclobutyl)amino]benzonitrile (3i). To a solution of 4-aminobenzonitrile (75 $\mathrm{mg}, 0.63 \mathrm{mmol})$ in $1.0 \mathrm{M} \mathrm{HCl} /$ ether $(1 \mathrm{ml})$, dichloromethane $(4 \mathrm{ml})$, THF $(5 \mathrm{ml})$ was added 1,2-bis (trimethylsilyloxy)cyclobutene $(121 \mathrm{mg}, 0.52 \mathrm{mmol})$ at $0^{\circ} \mathrm{C}$. The reaction mixture was heated at $80^{\circ} \mathrm{C}$ for 3.5 hours, then the solvent was removed under vacuum. Chromatography on silica gel eluting with $80 / 20$ ether/petroleum ether provided the 4cyano aniline cyclobutanone derivative $3 \mathbf{i}$ as yellow oil ( $25 \mathrm{mg}, 26 \%$ ). IR (thin film) $3583,2253,1790 \mathrm{~cm}^{-1} ;{ }^{1} \mathrm{H}$ NMR $\left(300 \mathrm{MHz}, \mathrm{CDCl}_{3}\right) \delta$ 7.48-7.46 $(2 \mathrm{H}, \mathrm{m}), 6.62-6.60(2 \mathrm{H}$, 
m), $4.85(2 \mathrm{H}, \mathrm{m}), 3.15(1 \mathrm{H}, \mathrm{m}), 3.02(1 \mathrm{H}, \mathrm{m}), 2.68-2.65(1 \mathrm{H}, \mathrm{m}), 1.88-1.85(1 \mathrm{H}, \mathrm{m}) ;{ }^{13} \mathrm{C}$ NMR (75 MHz, $\left.\mathrm{CDCl}_{3}\right) \delta 224.1,149.5,133.6,120.1,112.9,102.0,67.7,42.5,21.0 . \quad$ MS: $\mathrm{m} / \mathrm{z}=102\left(\mathrm{M}^{+}-84\right.$, loss of H-N-cyclobutanone $), \mathrm{m} / \mathrm{z}=187\left(\mathrm{M}^{+}+1\right)$.

Acknowledgements: NSF Grant DBI-0216630 is gratefully acknowledged for the Varian UNITY-300 NMR obtained through the NSF Biological Major Instrumentation Program, and the NSF REU Program (Research Experience for Undergraduates) is gratefully acknowledged for summer financial support for N.A.

\section{References}

1. Bellus, D.; Ernst, B. New synthetic methods. 71. Cyclobutanones and cyclobutenones in the synthesis of natural and synthetic products. Angewandte Chemie 1988, 100, 820850.

2. Namyslo, J. C.; Kaufmann, D. E. The Application of Cyclobutane Derivatives in Organic Synthesis. Chemical Reviews (Washington, DC, United States) 2003, 103, 14851537.

3. Lee-Ruff, E.; Mladenova, G. Enantiomerically Pure Cyclobutane Derivatives and Their Use in Organic Synthesis. Chemical Reviews (Washington, DC, United States) 2003, 103, 1449-1483.

4. Lowe, G.; Swain, S. Synthesis of a cyclobutanone analog of a b-lactam antibiotic. Journal of the Chemical Society, Perkin Transactions 1: Organic and Bio-Organic Chemistry (1972-1999) 1985, 391-398. 
5. Pauls, H. W.; Cheng, B.; Reid, L. S. 2-(Peptidamido)cyclobutanones: a novel strategy for the inhibition of serine elastases. Bioorg. Chem. 1992, 20, 124-134.

6. Fairlie, D. P.; Tyndall, J. D. A.; Reid, R. C.; Wong, A. K.; Abbenante, G.; Scanlon, M. J.; March, D. R.; Bergman, D. A.; Chai, C. L. L.; Burkett, B. A. Conformational Selection of Inhibitors and Substrates by Proteolytic Enzymes: Implications for Drug Design and Polypeptide Processing. J. Med. Chem. 2000, 43, 1271-1281.

7. Tyndall, J. D. A.; Nall, T.; Fairlie, D. P. Proteases universally recognize beta strands in their active sites. Chemical Reviews (Washington, DC, United States) 2005, 105, 973999.

8. Burgess, K.; Li, S.; Rebenspies, J. Chiral 1,3-cyclobutane amino acids: syntheses and extended conformations. Tetrahedron Lett. 1997, 38, 1681-1684.

9. Bursavich, M. G.; Rich, D. H. Designing Non-Peptide Peptidomimetics in the 21st Century: Inhibitors Targeting Conformational Ensembles. J. Med. Chem. 2002, 45, 541558.

10. Doyle, M. P.; Kundu, K.; Russell, A. E. Catalytic Addition Methods for the Synthesis of Functionalized Diazoacetoacetates and Application to the Construction of Highly Substituted Cyclobutanones. Org. Lett. 2005, 7, 5171-5174.

11. Lall, M. S.; Ramtohul, Y. K.; James, M. N. G.; Vederas, J. C. Serine and Threonine b-Lactones: A New Class of Hepatitis A Virus 3C Cysteine Proteinase Inhibitors. J. Org. Chem. 2002, 67, 1536-1547. 
12. Bisel, P.; Breitling, E.; Frahm, A. W. Diastereo- and enantioselective synthesis of (+)and (-)-cis-2-aminocyclobutanols. European Journal of Organic Chemistry 1998, 729733.

13. Overman, L. E.; Okazaki, M. E.; Jacobsen, E. J. Synthesis applications of cationic aza-Cope rearrangements. 16. Stereocontrolled synthesis of substituted ciscyclopenta[b]pyrrolidines. J. Org. Chem. 1985, 50, 2403-2405.

14. Heine, H. G.; Fischler, H. M. N,N-Disubstituted 2-aminocyclobutanones from 2hydroxycyclobutanones and secondary amines. Chem. Ber. 1972, 105, 975-981.

15. Seki, M.; Matsumoto, K. Synthesis of amino acids and related compounds. Part 43. A facile preparation of amino(diethoxyphosphoryl)acetic esters: transformation of phosphoranes to phosphonic esters. Synthesis 1996, 580-582.

16. Deshpande, S. R.; Likhite, A. P.; Rajappa, S. Transesterification of alkyl carbamate to aryl carbamate: effect of varying the alkyl group. Tetrahedron 1994, 50, 10367-10370. 


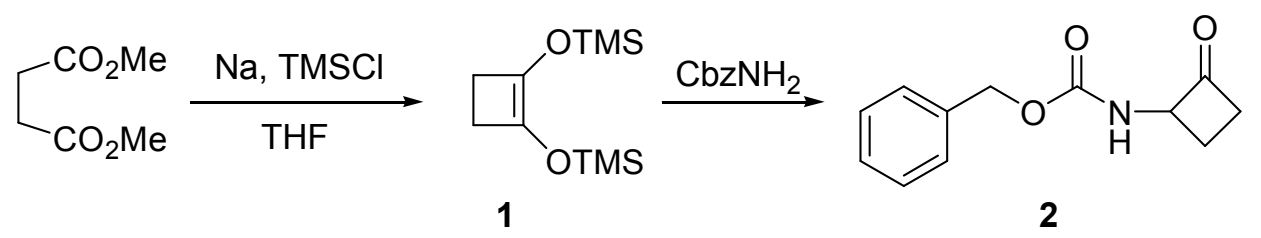

Scheme 1: Synthesis of benzyl (2-oxocyclobutyl)carbamate 
Table 1: Synthesis of 2-Aminocyclobutanone Derivatives 3a-i

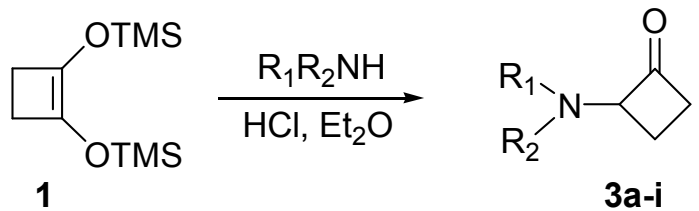

Entry




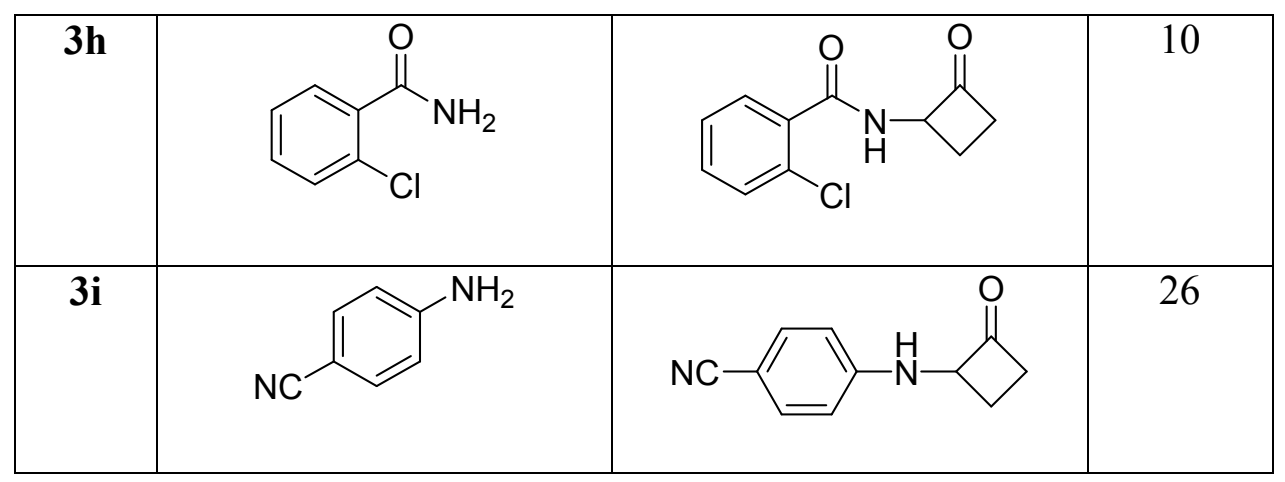

